\title{
The Evolution of Sediment Acoustic Models
}

\author{
Nicholas P. Chotiros and Marcia J. Isakson \\ Applied Research Laboratories, The University of Texas at Austin, \\ P. O. Box 8029, Austin, TX 78713-8029, email: chotiros@arlut.utexas.edu.
}

\begin{abstract}
Sediment acoustic models contain two connected components, the geo-physical description of the sediment and the model of acoustic processes. Geo-physical descriptors are used in the classification of sediments, and they are based on grain size, density and other physical descriptors. Acoustic sediment models were initially fluid approximations that were simple to implement. As the need for accuracy increased, the fluid model was extended to stratified fluid and visco-elastic models. The latter, with five frequency-independent parameters, appeared to be consistent with sediment acoustic data up to the 1980s. More recent experimental data have revealed discrepancies in the frequency-dependence of attenuation and sound speed, particularly in the case of sandy sediments, which cover a large fraction of the continental shelves. Broad-band acoustic measurements of wave speeds and attenuations are more consistent with a poro-elastic model, consisting of Biot's theory with extensions to account for the physics of granular media. Aside from terminology, there is a fundamental difference between viscoelastic and poro-elastic theories. The former is based on two types of waves, a compressional wave and a shear wave, while the latter has an additional compressional wave, often called the Biot wave. There are currently two approaches to the development of sediment acoustic models: (a) visco-elastic models with frequency dependent parameters that mimic the observed behavior, and (b) poro-elastic models that reflect the physical processes. It is shown that (a) would be a significant improvement over existing models, but (b) is the preferred solution.
\end{abstract}

Keywords: Seabed, transmission, reflection, propagation.

PACS: 43.30.Ma, 43.30.Pc, 43.30.Vh.

\section{INTRODUCTION}

Sediment classification based on grain size may be traced back to Wentworth [1], who divided sediments into classes according to their mean grain size. The classes were separated by multiples of 2 . A coarser classification into just 3 main categories: gravel, sand and mud was also put forward. Folk [2] and others sought to refine the classification scheme by considering mixtures of the three main categories. The terminology is still in use today. In addition, there are other physical properties that are important to underwater acoustics, such as density, porosity, and mechanical properties. We require a model that can take the geo-physical parameters as input and produce useful acoustical properties as the output, such as reflection coefficient, sound speed and absorption, as illustrated in figure 1. 


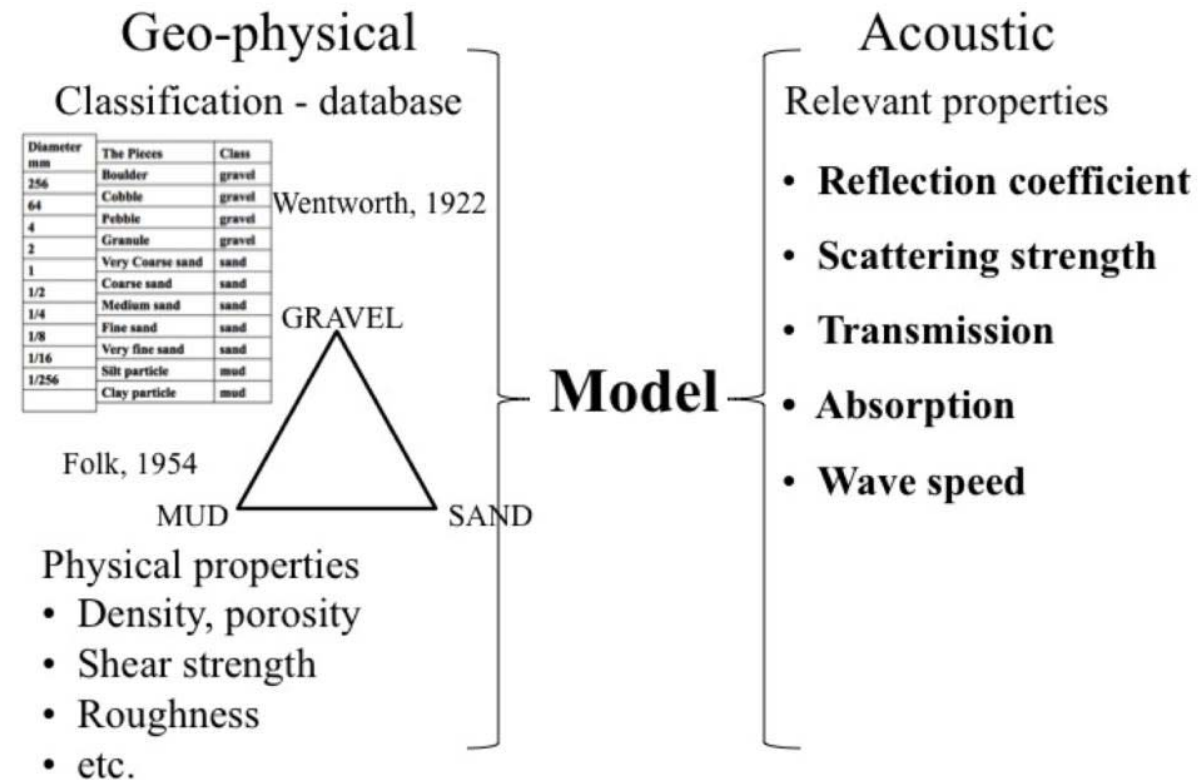

FIGURE 1. Illustration of the presumed connection between geo-physical and acoustic properties.

\section{EVOLUTION OF CURRENT MODELS}

The initial sediment models were fluid approximations, i.e. an incident wave from the water produces one transmitted wave in the sediment and one reflected wave in the water. The fluid model has three parameters: density, sound speed and loss tangent. Following the landmark investigation by Hamilton [3], visco-elastic models were adopted, in which an incident wave from the water produces two waves in the sediment, compressional and shear waves, and one reflected wave in the water. It has five parameters: the three from the fluid model plus two more, i.e. the shear wave speed and loss tangent. The model put forward by the Applied Physics Laboratory, University to Washington, also known as the High Frequency Environmental Acoustics (HFEVA) model, [4] followed this evolutionary path starting out in version 1.0 as a fluid model in 1994 and changing to a visco- elastic model with constant parameters in version 1.1. This is consistent with the experimental data from Hamilton and other investigators of the 1980s, as illustrated in figure 2. The compressional and shear wave speeds were considered to be independent of frequency, and dependent on density, as illustrated in the top right panel. The attenuation of both compressional and shear waves was assumed to increase linearly with frequency, as suggested by the data available at the time. 

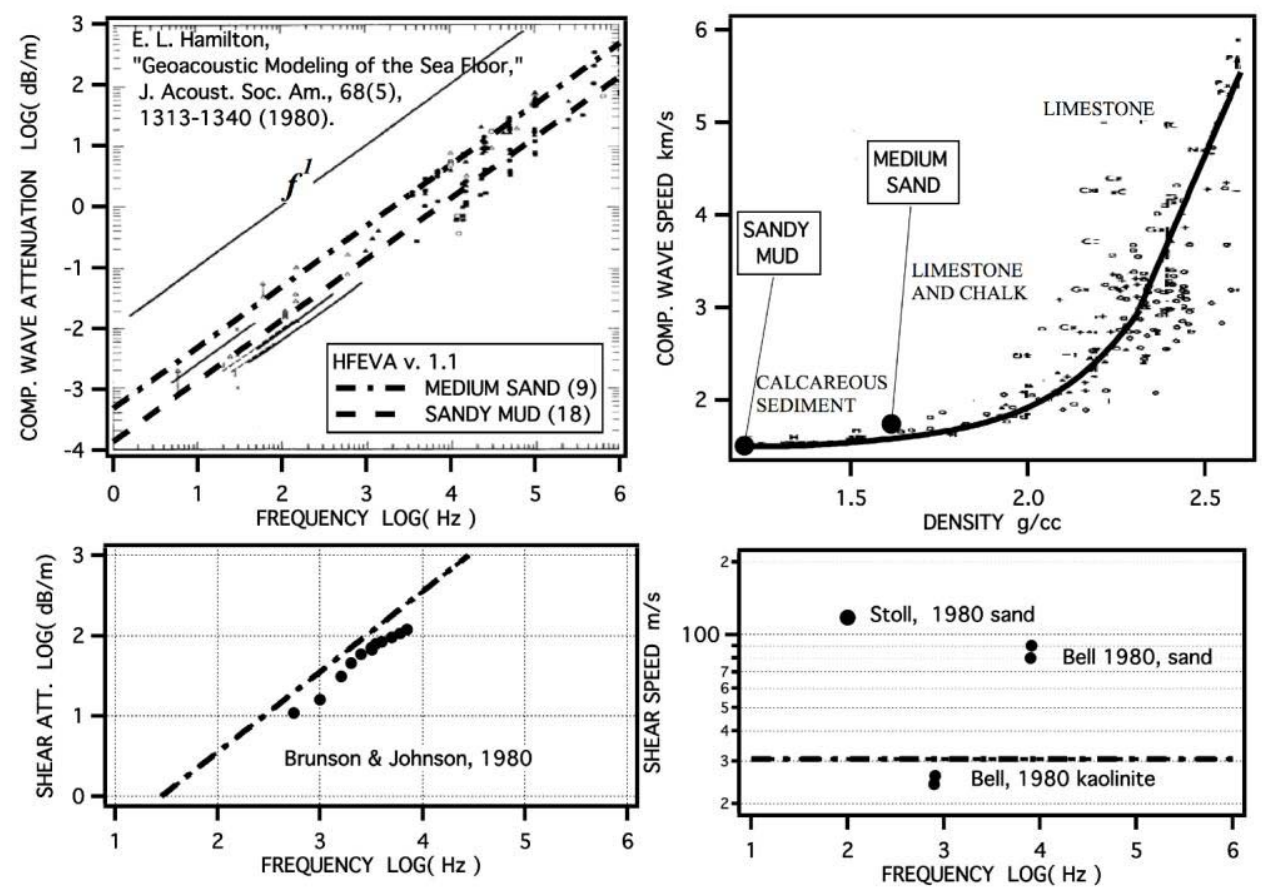

FIGURE 2. Published data from Hamilton 1980 and examples from the HFEVA.

\section{NEED FOR IMPROVEMENT}

More extensive measurements have been made since the 1980s, particularly the ONR sponsored experiments SAX99, ASIAEX, SAX04, and SW06. In the case of sandy sediments, it was found that the sound speed in sand changes with frequency, particularly in the 1 to $10 \mathrm{kHz}$ band. From direct measurements and inversions [5,6,7], it was found that attenuation increases approximately as the second power of the frequency, at frequencies below a few kiloHertz. At higher frequencies, the rate of increase is lower: in the case of laboratory sands it goes as the half power of frequency, but in natural sands it is often closer to the first power of frequency.

A visco-elastic model with constant parameters is inconsistent with the measurements, as shown in figure 3 . The Biot-Stoll poro-elastic model $[8,9,10]$ goes part of the way toward explaining the measurements, as shown in the same figure, but it cannot match the magnitude of the wave speed dispersion and high frequency attenuation trends.

A poro-elastic model, with modifications to account for the peculiarities of granular media such as sand[11], is a better fit to the measurements, as shown in figure 4 . This particular model is Biot + contact physics 2008 (BIC08). It includes the basic Biot equations, with modifications that account for the squirt flow at the grain contacts by Kimura [12], low frequency wave speed at low frequencies due to grain rotation [13], and high frequency attenuation and dispersion due to shear flow at the grain contacts [14]. 

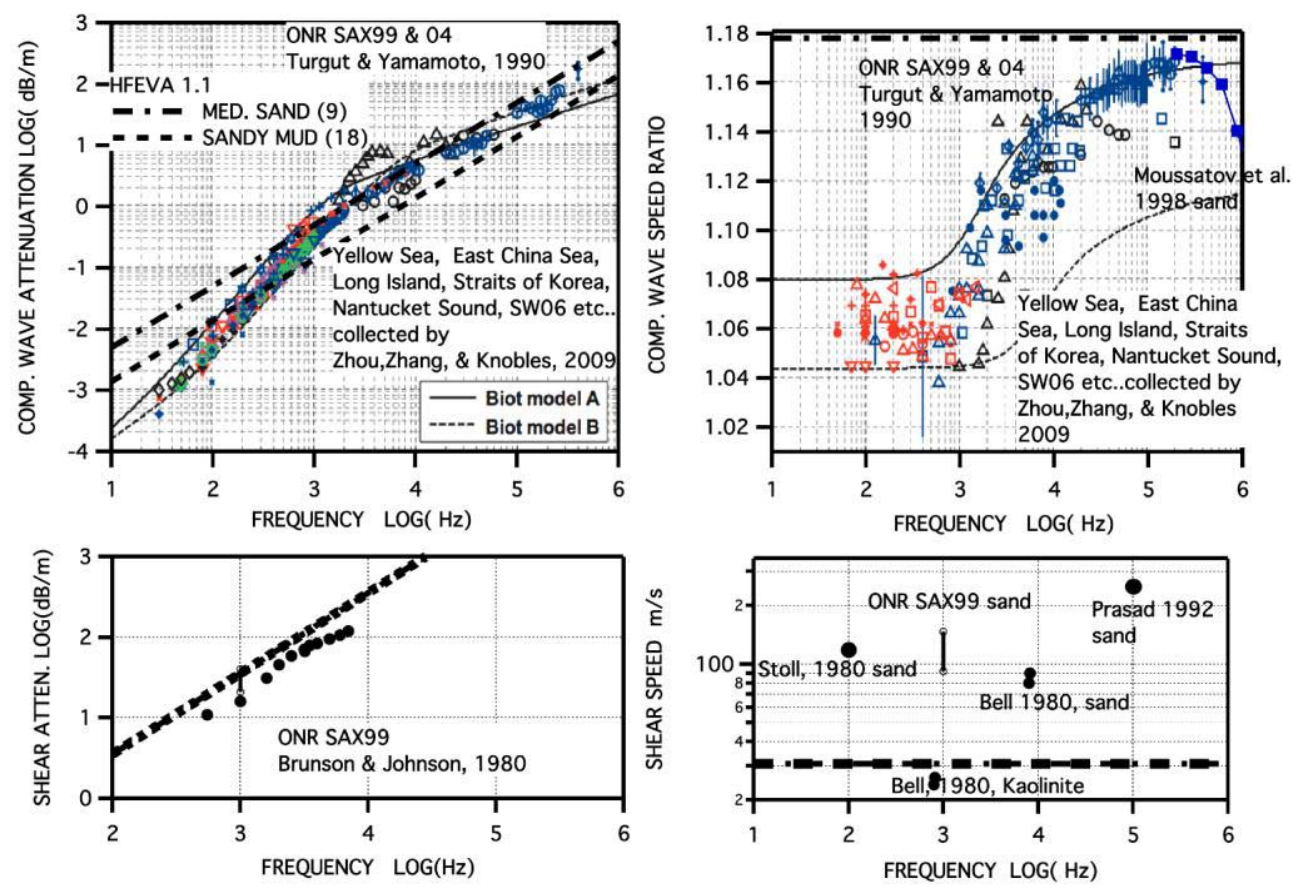

FIGURE 3. Recent measurements and inversions of wave speeds and attenuations in sandy sediments from numerous sources compared to visco-elastic models with constant parameters and with the Biot-Stoll model. The color scheme follows figures 9 and 10 in Ref. 5.
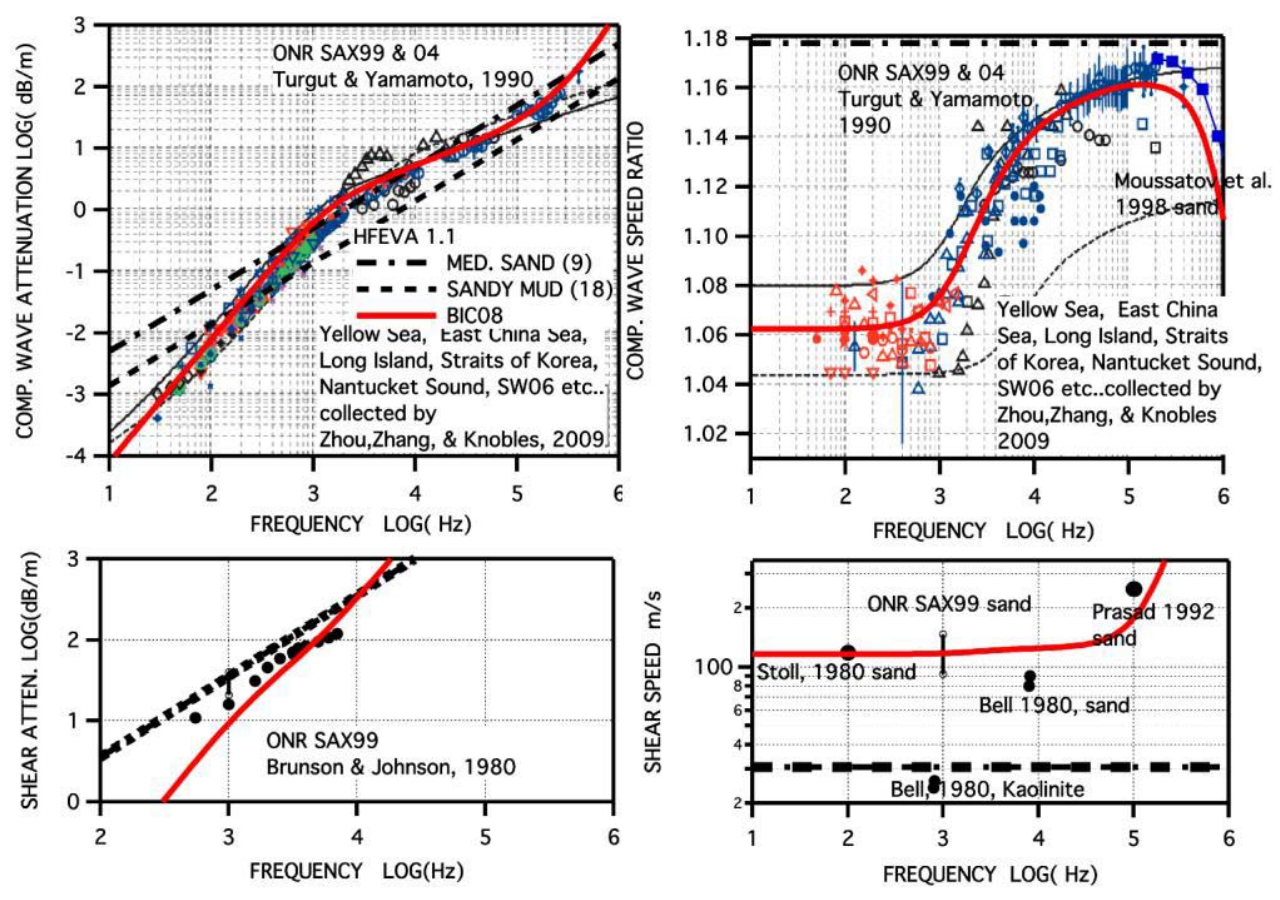

FIGURE 4. Recent measurements and inversions of wave speeds and attenuations in sandy sediments from numerous sources, and the BIC08 model. 
A direct comparison of a visco-elastic model with constant parameters and the BIC08 model, in terms of the reflection loss and transmission loss (TL) in a shallow water wave guide, illustrates the significance of the differences. The models were adjusted to have exactly the same wave speeds and attenuations at $2 \mathrm{kHz}$. Consider a TL calculation at this frequency in a simple waveguide, $50 \mathrm{~m}$ deep, with iso-velocity water and a uniform sediment bottom, with source and receiver at a depth of $4 \mathrm{~m}$.

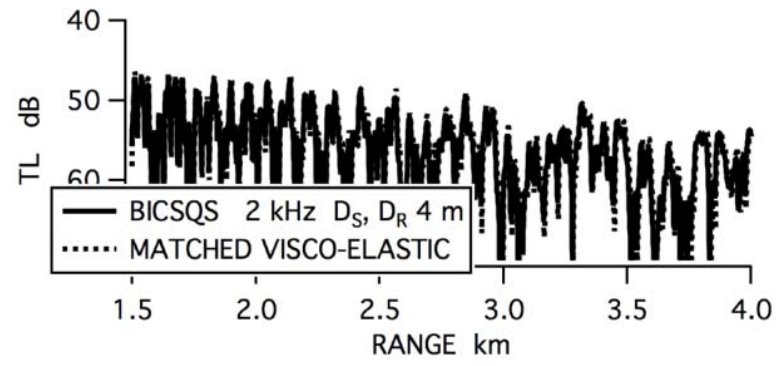

FIGURE 5. Comparison of the calculated TL of waveguides with visco-elastic and poro-elastic bottom moels, with matched wave speeds and attenuations at $2 \mathrm{kHz}$.

The computed TL curves for the two bottom models are compared in figure 5 . The difference is around $1 \mathrm{~dB}$ or less. In this case, it appears that the two models are approximately equivalent. The same models at $100 \mathrm{~Hz}$ show a very different picture, as illustrated in figure 6 . Since the wave speeds and attenuations change with frequency at different rates, the TL values differ by several decibels. This is a significant difference which deserves to be thoroughly researched.

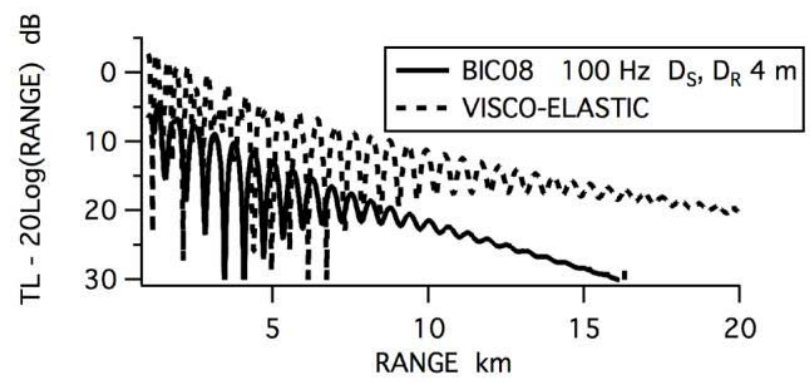

FIGURE 6. Comparison of the calculated TL in waveguides with visco-elastic and poro-elastic bottom models at $100 \mathrm{~Hz}$. The visco-elastic model has constant parameters that were matched to the poroelastic model at $2 \mathrm{kHz}$.

\section{SEDIMENT MODELS FOR THE FUTURE}

Models that are currently under development may be divided into two classes, (a) visco-elastic models with frequency dependent parameters and (b) poro-elastic models. These two classes of models are fundamentally different because in the poroelastic model, there are three waves transmitted into the sediment: two compressional waves and one shear wave, while in the visco-elastic models there is just one compressional wave in addition to the shear wave. 
The grain shearing (GS) models of Buckingham [15,16], the simplified Biot models of Pierce and Carey [17], and the Geoacoustic Bottom Interaction Model (GABIM) [18] all fall under the category of visco-elastic models with frequency dependent parameters. In this approach, the five parameters of a visco-elastic model are now considered to be frequency dependent, as illustrated by the example (solid curves) in figure 7.

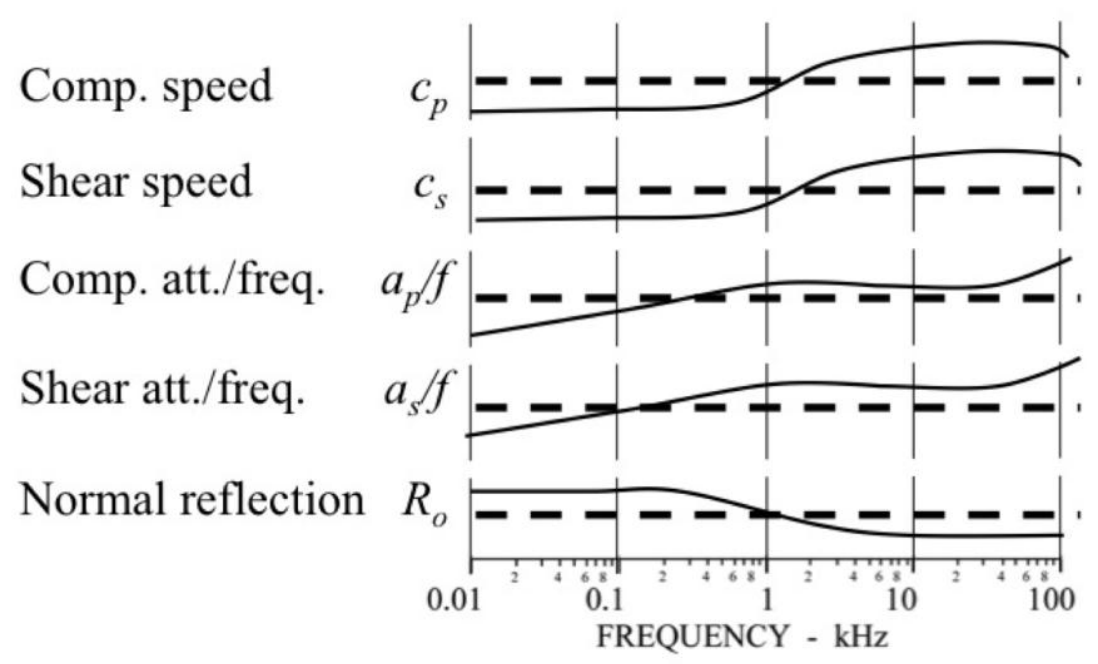

FIGURE 7. Illustration of a visco-elastic model with frequency dependent parameters.

A consequence of the difference between the two model categories is the effective density problem. Reflection loss, particularly at normal incidence is relatively simple to measure and it is used in sediment classification and mine burial prediction. The poro-elastic model is consistent with in-situ reflection loss measurements, but not the visco-elastic model. The difference between the two models is illustrated in figure 8 , which shows the predicted reflection loss at $2 \mathrm{kHz}$ when the parameter values are matched (solid and short dashed curves). In order to obtain the same reflection loss values, an artificially low value of sediment density, $11 \%$ lower than the true value, called the effective density, has to be used in the visco-elastic model (long dashed curve). The effective density is necessary to compensate for the absence of the Biot wave in the visco-elastic models. 


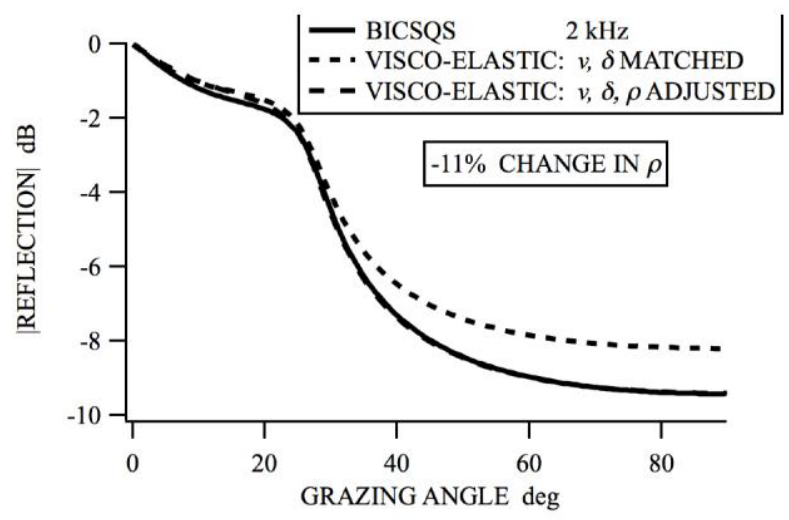

FIGURE 8. Comparison of reflection loss of visco-elastic and poro-elastic models with matched wave speeds and attenuations. It is necessary to use an artificial value of density to achieve the correct reflection loss in the visco-elastic model.

In the context of TL, it is noted, in the example of figure 5 , that the predicted TL curves are almost identical when the model parameters are matched. However, that is not always the case. This is illustrated by returning to the example in figure 6 , and matching the wave speeds and attenuations. The predicted TL values are closer, but significant differences remain, as shown in figure 9.

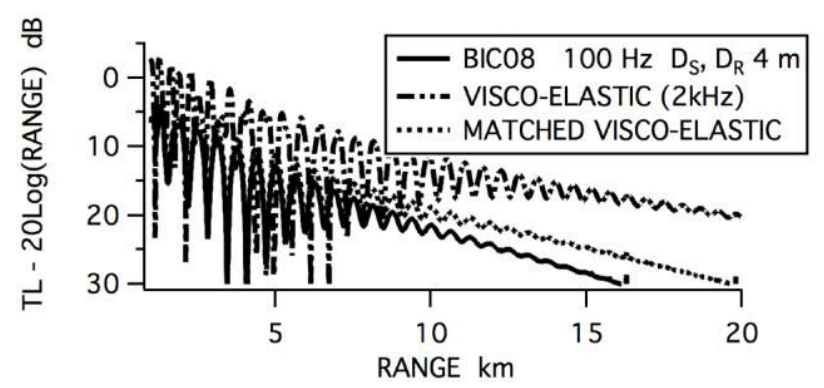

FIGURE 9. Comparison of the calculated TL of waveguides with visco-elastic and poro-elastic bottom models from Fig. 6, and a visco-elastic bottom model with matched wave speeds and attenuations at $100 \mathrm{~Hz}$.

The cause of the difference is the absence of the Biot wave in the elastic model, and the resulting differences in reflection loss. In figure 5 , at $2 \mathrm{kHz}$, there is very little difference because the dominant modes have very shallow grazing angles. In figure 6 , at $100 \mathrm{~Hz}$, the same modes have significantly steeper angles. The reflection loss curves in figure 8 show that the differences in reflection loss increase as the grazing angle increases.

Finally, the evolutionary time lines of the models may be summarized as illustrated in figure 10. The development of the two types of models, the visco-elastic models with frequency dependent parameters, and the poro-elastic models will likely continue in parallel, until practical and efficient formulations, with readily obtainable input parameters, are achieved. 


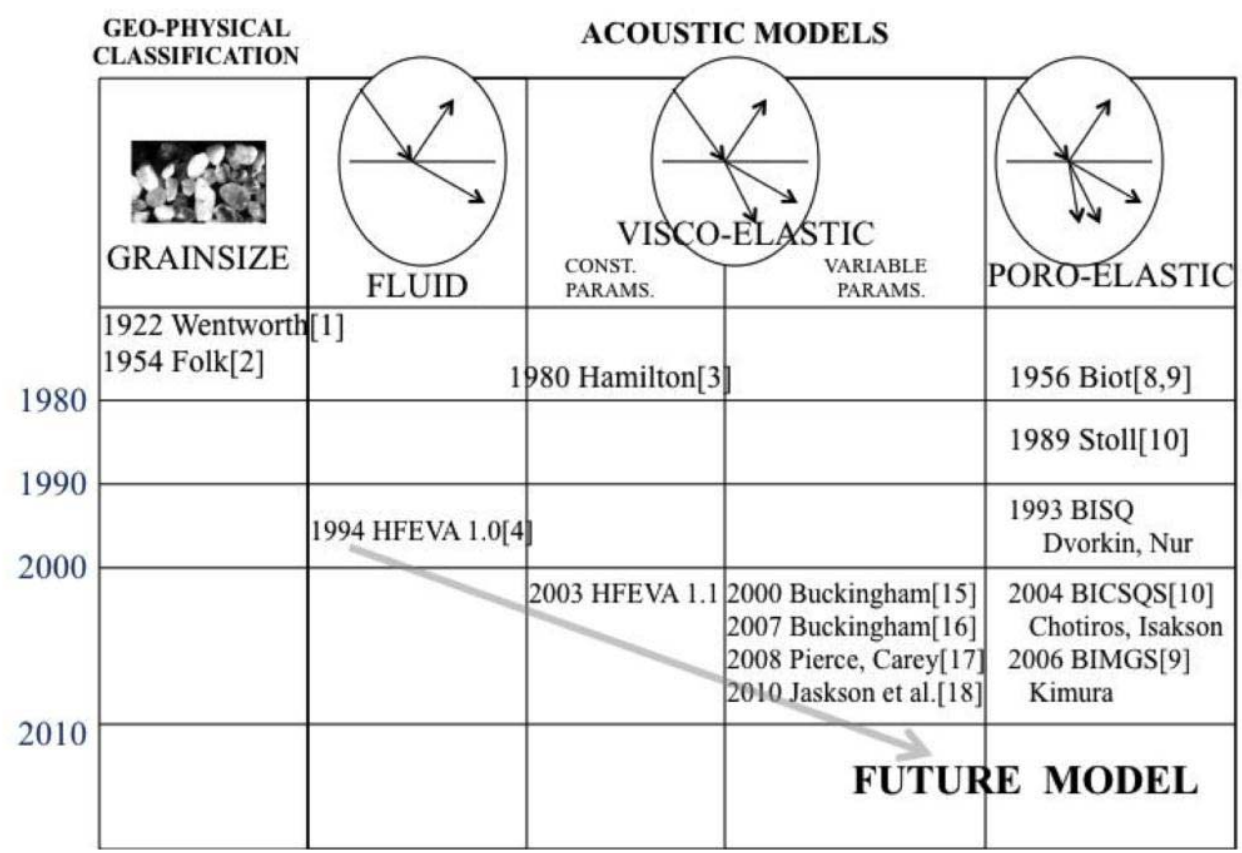

FIGURE 10. Time evolution of sediment classification and sediment acoustic models, showing the four main classes of models.

\section{ACKNOWLEDGMENTS}

This work was supported by the Office of Naval Research, Ocean Acoustics Program.

\section{REFERENCES}

1. C. K. Wentworth. "A scale of grade and class terms for clastic sediments," Journal of Geology 30, 5, 377-392, (1922).

2. R. L. Folk. "The distinction between grain size and mineral composition in sedimentary-rock nomenclature," Journal of Geology 62, 4, 344-359, (1954).

3. E. L. Hamilton. "Geoacoustic Modeling of the Sea Floor," J. Acoust. Soc. Am. 68, 5, 1313-1340, (1980).

4. APL-UW High-Frequency Ocean Environmental Acoustic Models Handbook, Applied Physics Laboratory, University of Washington, U.S. APL-UW 9407, October 1994.

5. J.-X. Zhou, X.-Z. Zhang, and D. P. Knobles. "Low-frequency geoacoustic model for the effective properties of sandy seabottoms," J. Acoust. Soc. Am. 125, 5, 2847-2866, (2009).

6. A. Turgut, and T. Yamamoto. "Measurements of acoustic wave velocities and attenuation in marine sediments," J. Acoust. Soc. Am. 87, 6, 2376-2382, (1990). 
7. K. L. Williams, D. R. Jackson, E. I. Thorsos, D. Tang, and S. G. Schock. "Comparison of sound speed and attenuation measured in a sandy sediment to predictions based on the Biot Theory of porous media," IEEE J. Oceanic Eng. 27, 3, 413-428, (2002).

8. M. A. Biot. "Theory of Propagation of Elastic Waves in a Fluid-Saturated Porous Solid -- I. Low Frequency Range," J. Acoust. Soc. Am. 28, 2, 168-178, (1956).

9. M. A. Biot. "Theory of Propagation of Elastic Waves in a Fluid-Saturated Porous Solid -- II. Higher Frequency Range," J. Acoust. Soc. Am. 28, 179-191, (1956).

10. R. D. Stoll, Sediment acoustics. (Springer-Verlag, New York, 1989).

11. N. P. Chotiros, and M. J. Isakson. "A broadband model of sandy ocean sediments: Biot-Stoll with contact squirt flow and shear drag," J. Acoust. Soc. Am. 116, 4, 2011-2022, (2004).

12. M. Kimura. "Frame bulk modulus of porous granular marine sediments," J. Acoust. Soc. Am. 120, 2 , 699-710, (2006).

13. N. P. Chotiros, and M. J. Isakson. "Acoustic virtual mass of granular media," J. Acoust. Soc. Am. 121, 2, EL70-EL76, (2007).

14. N. P. Chotiros, and M. J. Isakson. "High-frequency dispersion from viscous drag at the grain-grain contact in water-saturated sand," J. Acoust. Soc. Am. 124, 5, EL296-301, (2008).

15. M. J. Buckingham. "Wave propagation, stress relaxation, and grain-to-grain shearing in saturated, unconsolidated marine sediments," J. Acoust. Soc. Am. 108, 6, 2796-2815, (2000).

16. M. J. Buckingham. "On pore-fluid viscosity and the wave properties of saturated granular materials including marine sediments," J. Acoust. Soc. Am. 122, 3, 1486-1501, (2007).

17. A. D. Pierce, and W. M. Carey. "Low-frequency attenuation of acoustic waves in sandy/silty marine sediments," J. Acoust. Soc. Am. 124, 5, EL308 - EL312, (2008).

5. D. R. Jackson, R. I. Odom, M. L. Boyd, and A. N. Ivakin. "A Geoacoustic Bottom Interaction Model (GABIM)," IEEE J. Oceanic Eng. 35, 3, 603-617, (2010). 
Copyright of AIP Conference Proceedings is the property of American Institute of Physics and its content may not be copied or emailed to multiple sites or posted to a listserv without the copyright holder's express written permission. However, users may print, download, or email articles for individual use. 